\title{
Procedure for verification of sound source coverage over façades according to the International Standard ISO 140-5
}

\author{
José Luis Sánchez Bote , Antonio Pedrero González, Juan José Gómez Alfageme \\ DIAC-E.U.I.T. Telecomunicación, Universidad Politécnica de Madrid, Ctra. Valencia, km 7, 28031 Madrid, Spain
}

\begin{abstract}
A B S T R A C T
This paper presents a new verification procedure for sound source coverage according to ISO $140-5$ requirements. The ISO 140-5 standard applies to the measurement of façade insulation and requires a sound source able to achieve a sufficiently uniform sound field in free field conditions on the façade under study. The proposed method involves the electroacoustic characterisation of the sound source in laboratory free field conditions (anechoic room) and the subsequent prediction by computer simulation of the sound free field radiated on a rectangular surface equal in size to the façade being measured. The loudspeaker is characterised in an anechoic room under laboratory controlled conditions, carefully measuring directivity, and then a computer model is designed to calculate the acoustic free field coverage for different loudspeaker positions and façade sizes. For each sound source position, the method provides the maximum direct acoustic level differences on a façade specimen and therefore determines whether the loudspeaker verifies the maximum allowed level difference of $5 \mathrm{~dB}$ (or $10 \mathrm{~dB}$ for façade dimensions greater than $5 \mathrm{~m}$ ) required by the ISO standard. Additionally, the maximum horizontal dimension of the façade meeting the standard is calculated and provided for each sound source position, both with the $5 \mathrm{~dB}$ and $10 \mathrm{~dB}$ criteria. In the last section of the paper, the proposed procedure is compared with another method used by the authors in the past to achieve the same purpose: in situ outdoor measurements attempting to recreate free field conditions. From this comparison, it is concluded that the proposed method is able to reproduce the actual measurements with high accuracy, for example, the ground reflection effect, at least at low frequencies, which is difficult to avoid in the outdoor measurement method, and it is fully eliminated with the proposed method to achieve the free field requisite.
\end{abstract}

\section{Introduction}

Section 4.2 of the International Standard ISO $140-5$, "Field measurements of airborne sound insulation of façade elements and façades" [1-3], specifies that the directivity of the loudspeaker used in a test must ensure local level differences less than $5 \mathrm{~dB}$ (or $10 \mathrm{~dB}$ for façade dimensions greater than $5 \mathrm{~m}$ ), as measured in a free field over an area the same size and orientation as the wall or element to be tested. This requirement must be verified in the frequency bands of interest defined as, at a minimum, the third octave bands from $100 \mathrm{~Hz}$ to $3150 \mathrm{~Hz}$, and preferably from $50 \mathrm{~Hz}$ to $5 \mathrm{kHz}$. In addition, the ISO/IEC 17025 standard, "General requirements for the competence of testing and calibration laboratories", in Section 5.5.2 about equipment [4], requires accredited laboratories to validate their equipment compliance with all technical requirements of the testing standards, and compliance testing should be performed periodically if the equipment is susceptible to changing its characteristics over time.

As stated in the ISO 140-5 standard, the uniformity level condition of the façade must be measured in free field conditions, but the verification is difficult to perform in actual conditions because reflections from nearby surfaces are difficult to avoid. In recent years, the authors have performed multiple insulation measurements of building façades according to the ISO 140-5 standard and, before development of the procedure described in this paper, the verification of the sound source coverage on the specimen surface under study has always been a prohibitively expensive task, providing neither robust nor repeatable results. In those cases, the coverage specification was measured on an imaginary surface having the same dimensions as the façade being tested, and situated in an outside location, with ground sound reflection always present and too great to produce a sound free field on the surface under consideration.

Accounting for the abovementioned difficulties, the authors have recently implemented a method at the Laboratory of Acoustics of the EUIT Telecomunicación (Universidad Politécnica de Madrid) in which the direct sound field on a surface, in the layout 
indicated in the ISO standard, is calculated from directivity measurements of loudspeakers performed in an anechoic room [5] and used to verify the sound source.

Some other strategies exist to verify the coverage of a sound source on a façade in an anechoic room. For example, verification can occur through the actual geometry, which would require a large (and usually infeasible) anechoic room, or through scaleddown geometry, where small inaccuracies in the microphone positions can generate large errors in the results. There is no flexibility in these two procedures to extend the findings to other surface sizes or loudspeaker positions.

The verification procedure developed in this study has two parts. The first part includes the measurement and characterisation of the sound source. In the second part, calculations and predictions are made for the specified façade. Section 2 describes the measurement stage, performed over the loudspeaker in free field conditions (i.e., an anechoic room) to determine sound directivity in the horizontal and vertical planes. The frequency response of the source is also measured. Section 3 focuses on the prediction step that consists of estimating the direct sound field on an imaginary surface identical to that described in ISO $140-5$ and with variable dimensions. If no specific façade size is required, the sound level estimation will be performed on the size considered standard in the publication, a surface having the horizontal and vertical dimensions $\Delta x \times \Delta y=4 \mathrm{~m} \times 3 \mathrm{~m}$. In addition, the procedure includes the estimation of the maximum horizontal dimension $\Delta x_{\max }$ of the rectangular façade that, with a variable aspect ratio of $\Delta x /$ $\Delta y=1,4 / 3,3 / 2$ and 2 , verifies either the $5 \mathrm{~dB}$ or $10 \mathrm{~dB}$ criterion of the ISO standard relative to the maximum direct sound level differences allowed on the façade. Finally, Section 4 compares the presented coverage verification procedure, based on computer prediction, with the older method, based on in situ outdoors measurements in acoustic free field conditions, used by the authors in the past.

\section{Measurements of the sound source in an anechoic room}

\subsection{Measurement scenario}

The measurements over the sound source are performed in the anechoic room of the Laboratory of Acoustics of the EUIT Telecomunicación (Universidad Politécnica de Madrid). Pictures of the measurement scenario corresponding to a test case are shown in Fig. 1. The sound source is placed on a diagonal of the anechoic room, $1.5 \mathrm{~m}$ from the absorbent wedges of one of the corners of the room and with its acoustic centre at height $h=1.25 \mathrm{~m}$ and $h=1 \mathrm{~m}$ above the metallic grid floor to measure horizontal and vertical directivity, respectively (see the arrangement scheme in Fig. 2). Although the electroacoustic characteristics of the sound source should not depend on distance, measurements are made at $1 \mathrm{~m}$ and $1.5 \mathrm{~m}$ to check the measurement's repeatability and the anechoic properties of the room.

\subsection{Procedure for frequency response and horizontal-vertical directivity measurement in the $1 / 3$ octave bands from $100 \mathrm{~Hz}$ to $5 \mathrm{kHz}$}

Among the different methods available to perform the necessary measurements, the method based on pink noise excitation of the sound source was chosen due to equipment availability, although any of the usual methods from the literature are applicable. The measurement recording and initial data processing is performed with the B\&K PULSE 3560 spectrum analyser.

The sound source is excited via a power amplifier with a pink noise signal, and its acoustic output is picked up by the measurement microphone connected to the spectrum analyser. The acoustic spectrum is measured in one-third octave bands in different rotation positions of the speaker, covering a $360^{\circ}$ circle on both the horizontal and vertical planes, with an accuracy of $2.5^{\circ}$. The flat pink noise excitation spectrum (electrical signal) is also recorded simultaneously at identical points. From these measurements, the electroacoustic transfer function $\mathrm{SPL} / 2.83 \mathrm{~V}[\mathrm{~dB} / \mathrm{W}]$ (conventional voltage for speakers, representing the electric power $1 \mathrm{~W} /$ $8 \Omega$ ) is calculated for each point in relation to a $1-\mathrm{m}$ distance, independently of the real distance measured (Fig. 3). Using the spectra measured at each point, the horizontal and vertical directivity functions, $D_{H}(\theta)[\mathrm{dB}]$ and $D_{V}(\varphi)[\mathrm{dB}]$, respectively, are calculated for each frequency band (Fig. 4).

\section{Calculations and predictions of the sound source being studied based on the electroacoustic measurements}

\subsection{Loudspeaker simulation using electroacoustic software}

The data obtained following the procedure described in Section 0 characterise the sound source electroacoustically, both in directivity and in frequency response, and can be used to predict the loudspeaker behaviour over the façade specimen according to the ISO 140-5 standard. The geometry of the façade insulation test prescribed by the ISO standard is described in detail in [6]. Although this geometric information, along with the loudspeaker characterisation, may be enough to perform predictions, the authors have used the EASE software tool [7-9] to implement sound source verification in the method proposed here. Any other computer tool can be used instead of EASE if the information from
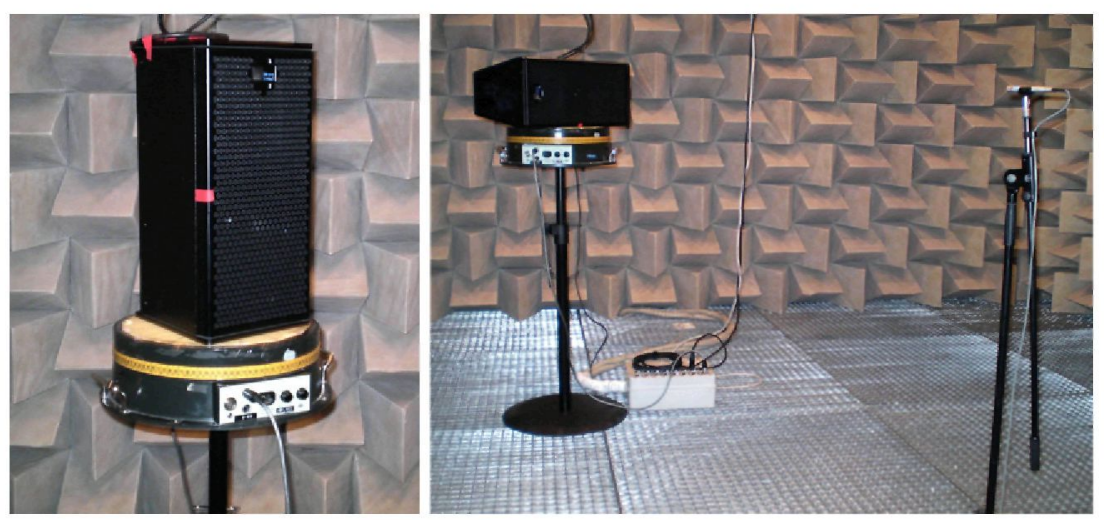

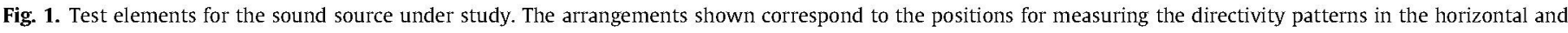
vertical planes. 

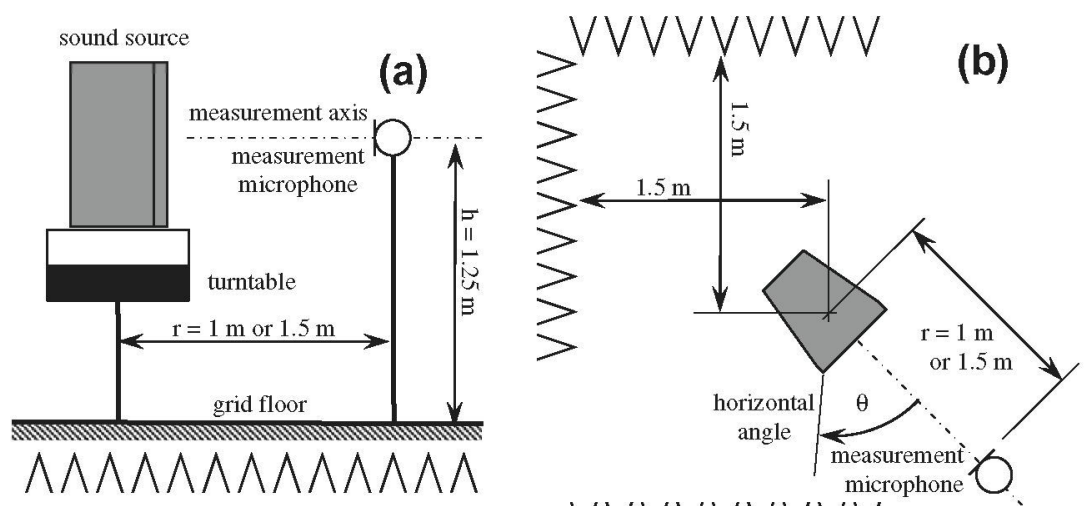

(c)
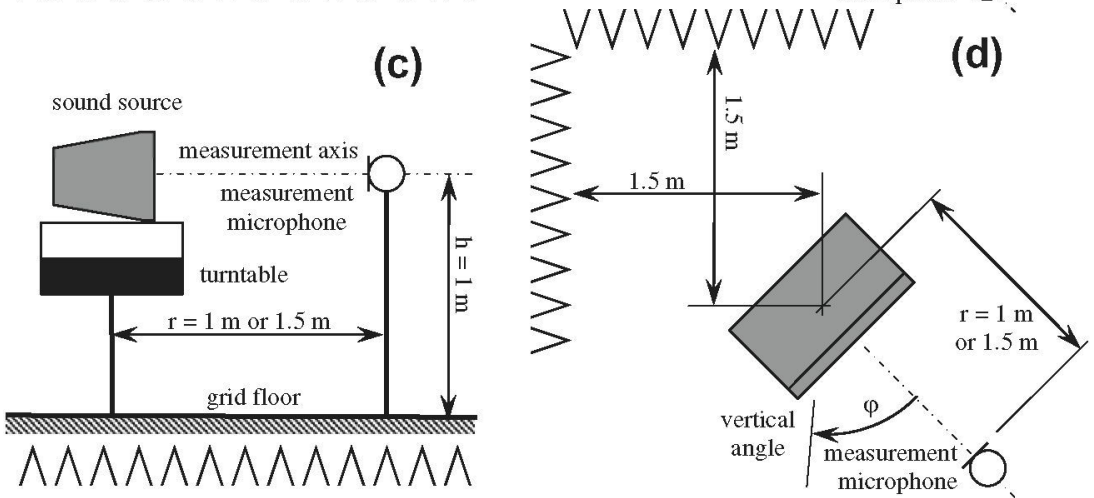

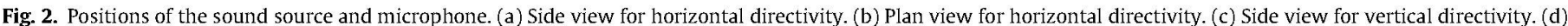
Plan view for vertical directivity.
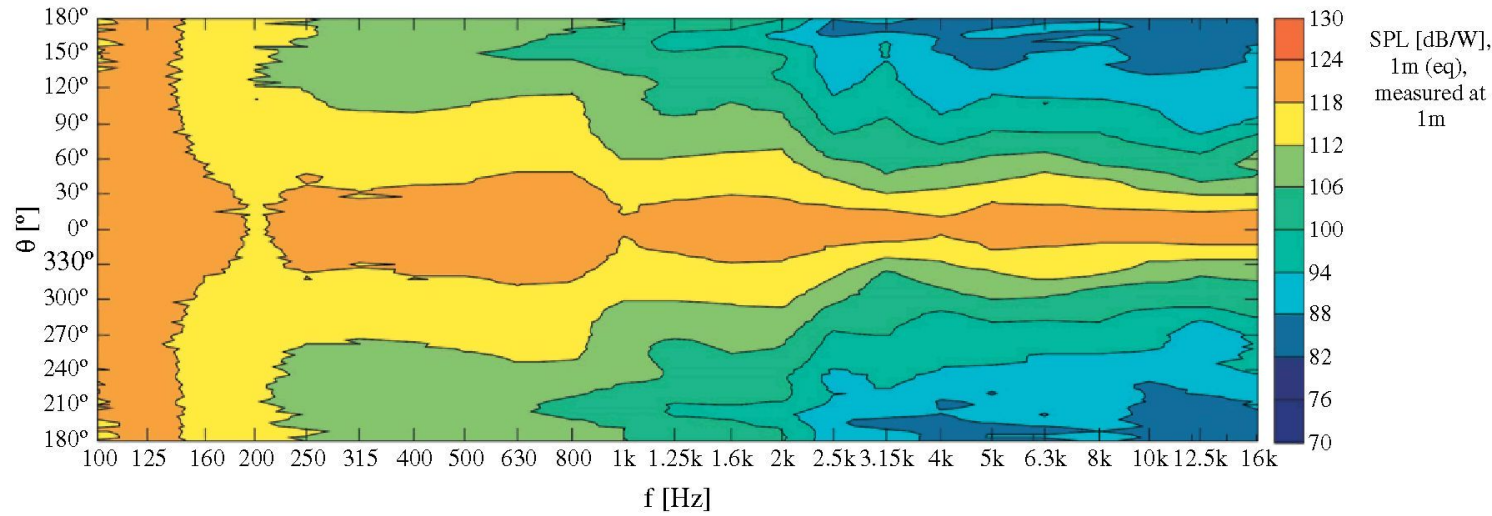

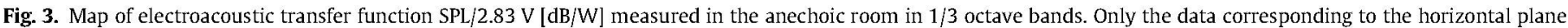
are shown.

[6] is applied. The data, in the form of one-third octave bands, have been introduced in the EASE component "EaseSpkr" to generate a new loudspeaker model (Fig. 5) of the actual sound source measured in anechoic room.

The accuracy required for directivity data in EASE is $5^{\circ}$. A downsampling of the data is required because the directivity measurements were made with accuracy of $2.5^{\circ}$. Measuring directivity in the horizontal and vertical planes may be sufficient for sound sources with cylindrical symmetry because the intermediate planes can be interpolated with accuracy. This procedure is normally used with the method described herein because the sound sources used for measuring the insulation on façades usually have approximately cylindrical symmetry. This assumption is not applicable to other cases, and measuring the directivity patterns in the intermediate planes with the required accuracy of at least $5^{\circ}$ is mandatory. In EASE, the interpolation of the intermediate planes can be performed using the software's own method called "elliptic lobe". Fig. 6 shows an example of horizontal-vertical polar plots in EASE after data input at the $1 / 3$ octave band of $1 \mathrm{kHz}$ and directivity globes after plane interpolation using the elliptic lobe.

\subsection{Prediction of the direct sound field distribution on a surface according to the ISO 140-5 standard}

Using the computer-aided design tool, the model of the loudspeaker under study was placed in a scenario described by the ISO $140-5$ standard. A calculation surface with the standard façade dimensions of $4 \mathrm{~m} \times 3 \mathrm{~m}$ is modelled. The loudspeaker is aimed at the centre of the façade (not mandatory in the ISO standard) in such a way that its position has an angle of $45^{\circ}$ with the surface, 

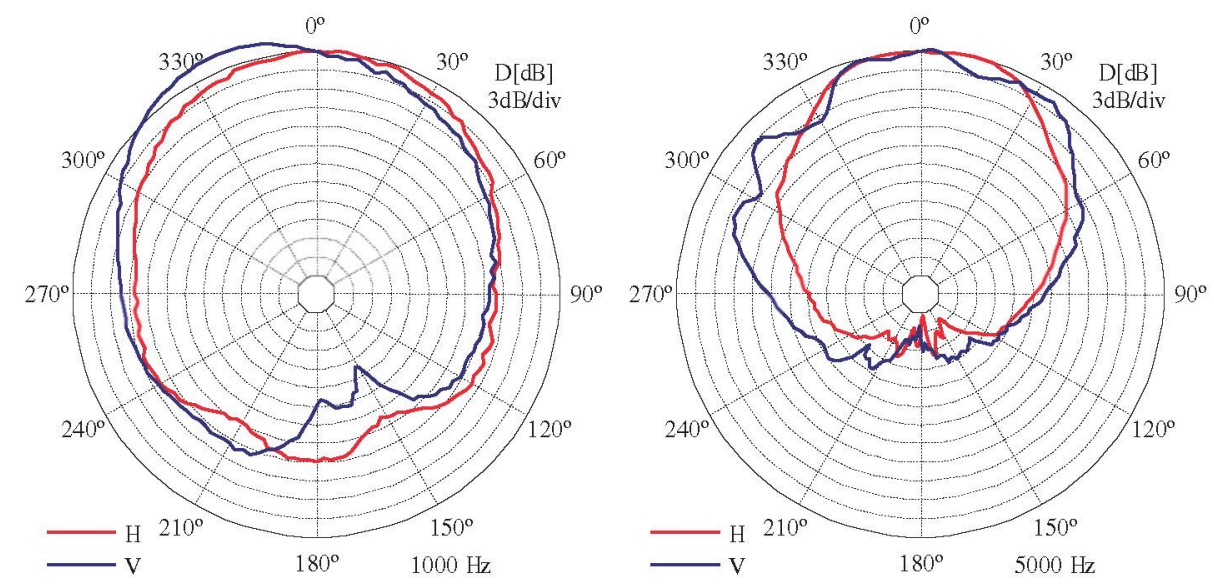

Fig. 4. Horizontal and vertical polar plots, $D_{H}(\theta)[\mathrm{dB}]$ and $D_{V}(\phi)[\mathrm{dB}]$, measured in the anechoic room. The $1 \mathrm{kHz}$ and $5 \mathrm{kHz} 1 / 3$ octave bands are shown as an example.

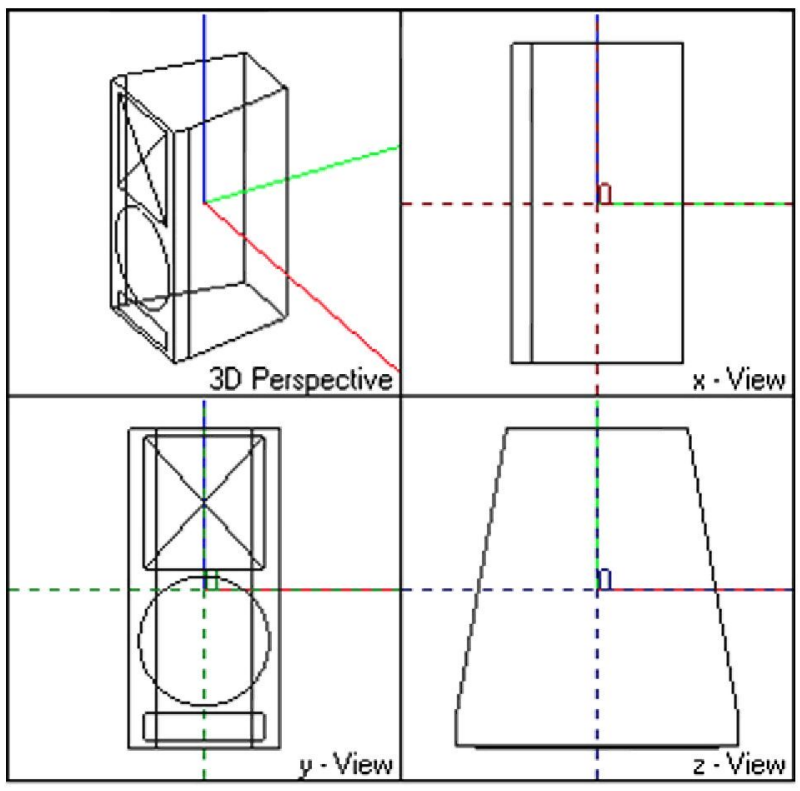

Fig. 5. Speaker model designed with the loudspeaker software. The acoustic centre of the loudspeaker is placed at the origin.

as stated in the ISO standard, and a distance of $5 \mathrm{~m}$ from the façade plane (Fig. 7). This is the shortest distance to the façade allowed by the ISO standard, and it produces more level differences on the surface. As stated in [6], for each façade size, or more precisely for each aspect ratio $\Delta x / \Delta y$ of its surface, there are two characteristics positions of the loudspeaker to be tested: the "most favourable" position and the "least favourable" one. The "most favourable" position corresponds a priori to that position in which the loudspeaker is placed just below the centre of the facade with an azimuth angle in spherical coordinates of $\phi_{\alpha^{+}}=-90^{\circ}$. On the other hand, the "least favourable" position has the sound source located on the façade diagonal with an azimuth angle of $\phi_{\alpha_{-}}=-36.9^{\circ}$ (for the case of $4 \mathrm{~m} \times 3 \mathrm{~m}$ dimensions). In both cases, the emitter is placed horizontally (as if standing on the ground), simulating its position in real tests.

The direct sound pressure level emitted from the previously modelled sound source is calculated over the $4 \mathrm{~m} \times 3 \mathrm{~m}$ surface using the computer model. The loudspeaker is driven by a flat spectrum signal (pink noise type). The chosen calculation accuracy is $4 \mathrm{~cm}$, corresponding to $\lambda / 8$ at $1 \mathrm{kHz}$. "Isoline" type coverage maps are obtained and represented, and the histogram of the level distribution on the surface at each frequency band is considered. The calculations take into account the sound absorption in the air due to its physical conditions, which include relative humidity of $60 \%$, temperature of $20^{\circ} \mathrm{C}$ and static atmospheric pressure of $1013 \mathrm{hPa}$. A sampling of results is shown in Fig. 8.

The final goal of the verification method presented here is to determine whether the modelled loudspeaker meets the level uniformity specifications of the ISO standard with respect to the façade under study. Consequently, the maximum variation of direct sound level on the surface must be estimated. The process followed thus far gives the results shown in Fig. 9, in which the maximum level differences in the one-third octave bands from $100 \mathrm{~Hz}$ to $5 \mathrm{kHz}$ over the $4 \mathrm{~m} \times 3 \mathrm{~m}$ façade are visualised. The results correspond to two loudspeaker positions, $S_{+}\left(-90^{\circ}\right)$ and $S_{-}\left(-36.9^{\circ}\right)$, otherwise known as the "most favourable" and "least favourable" emission points. The final verdict of the verification process is whether the local level differences are less than $5 \mathrm{~dB}$ (or $10 \mathrm{~dB}$ for façade dimensions greater than $5 \mathrm{~m}$ ), and therefore whether the limits of $\triangle \mathrm{SPL}=5 \mathrm{~dB}$ or $\triangle \mathrm{SPL}=10 \mathrm{~dB}$ shown in Fig. 9 are exceeded in the test results.

Although the results obtained by this procedure may be sufficient for verification, they are limited by the validity of a sound source for a particular façade size, in this case for a size of $4 \mathrm{~m} \times 3 \mathrm{~m}$. It would be interesting to generalise the study for any façade size. The maximum façade size that meets the ISO standard criteria, either with $\triangle \mathrm{SPL}=5 \mathrm{~dB}$ or $\triangle \mathrm{SPL}=10 \mathrm{~dB}$, could be studied using the same computer model. This information can be extracted by studying the direct sound field produced by the modelled loudspeaker on a large surface. The surface size is then progressively reduced until the level uniformity required by the ISO standard is met. Fig. 10 shows the maximum horizontal dimension of the façade $\Delta x_{\max }$ that could be used to verify ISO $140-5$, with either $\triangle \mathrm{SPL}=5 \mathrm{~dB}$ or $\triangle \mathrm{SPL}=10 \mathrm{~dB}$, considering one-third octave bands up to $5 \mathrm{kHz}$ and shown compared to the façade aspect ratio $\Delta x /$ $\Delta y$. To obtain this graph, the direct level distribution on a square façade measuring $22 \mathrm{~m} \times 22 \mathrm{~m}$ has been analysed with an accuracy of $5 \mathrm{~cm}$. Four aspect ratios representative of actual building walls have been tested: $\Delta x / \Delta y=1,4 / 3,3 / 2$ and 2 . Two loudspeaker positions have been analysed for the two crucial positions, "most favourable" (azimuth angle $\phi_{\alpha^{+}}=-90^{\circ}$ ) and "least favourable" (azimuth angles $\phi_{\alpha_{-}}=-45^{\circ},-36.9^{\circ},-33.7^{\circ}$ and $-26.6^{\circ}$, respectively for $\Delta x / \Delta y=1,4 / 3,3 / 2$ and 2 ). In all cases, the loudspeaker is placed horizontally $5 \mathrm{~m}$ from the facade and aimed at its centre. The maximum dimensions obtained with a reference omnidirectional loudspeaker called a "sphere" are also represented in this figure. 

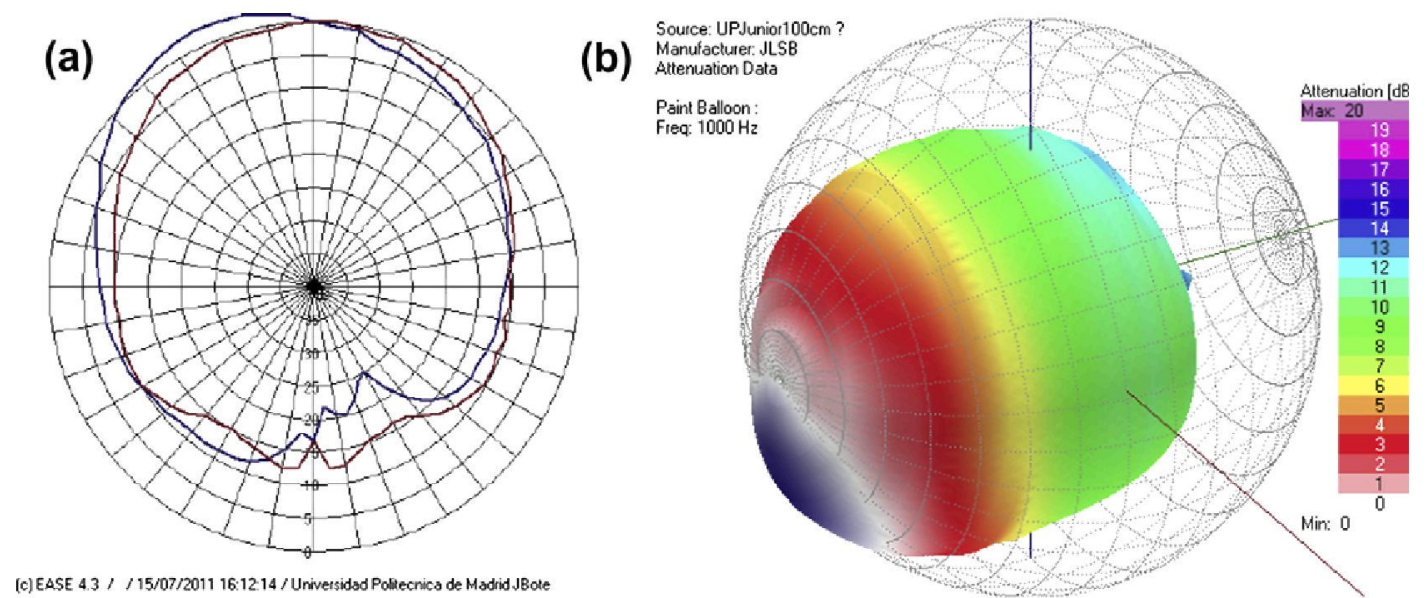

(c) EASE 4.3 / EASE Hall / 15/07/2011 16:16:19/Universidad Politecnica de Madrid JBote

Fig. 6. Example of directivity representations in the loudspeaker software after data input (frequency band $1 \mathrm{kHz}$ ). (a) Horizontal and vertical directivity. (b) Directivity globe after plane interpolation using the software's "elliptic lobe" method.

Table 1 shows the maximum horizontal dimensions $\Delta x_{\max }[\mathrm{m}]$ extracted from Fig. 10 for the case of the sound source previously modelled and projected over the $22 \mathrm{~m} \times 22 \mathrm{~m}$ façade.

\section{Comparison between the coverage verification procedure based on computer prediction and the method based on in situ outdoor measurements in acoustic free field conditions}

The authors found many problems in the verification of loudspeaker properties when they performed measurements of the airborne façade sound insulation of actual buildings according to the ISO $140-5$ standard. Before the implementation of the procedure described in this paper, the coverage uniformity of the sound source was tested outdoors with a setting as close as possible to an acoustic free field, measuring the sound levels produced by the source over a fictitious surface whose arrangement was equal to the in situ measurement of the final insulation test. This section compares loudspeaker verifications made previously by the

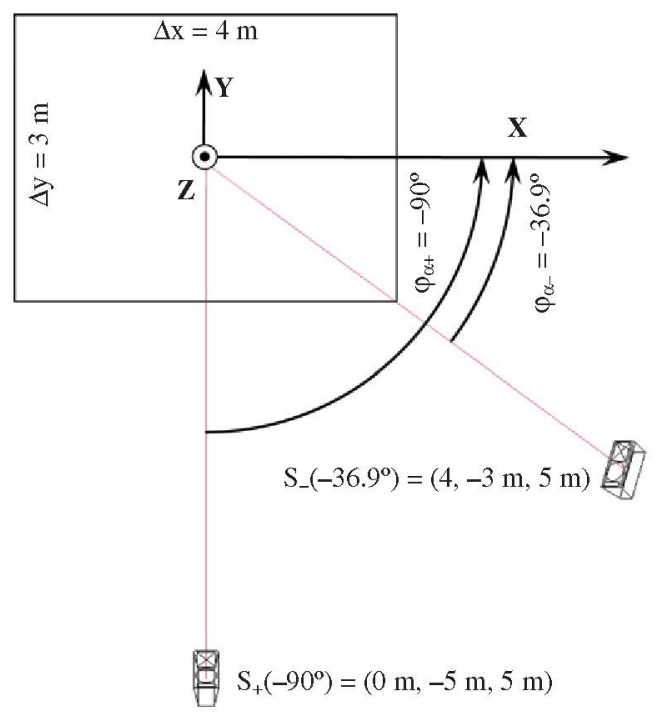

Fig. 7. Plan view in the EASE coordinate system of two emitting points with the sound source at $5 \mathrm{~m}$ from the façade of size $\Delta x \times \Delta y=4 \mathrm{~m} \times 3 \mathrm{~m}$. These two positions, fully described in [6], are called the "most favourable" and "least favourable" positions: $S_{+}\left(-90^{\circ}\right)=(0 \mathrm{~m},-5 \mathrm{~m}, 5 \mathrm{~m})$ and $\mathrm{S}_{-}\left(-36.9^{\circ}\right)=(4 \mathrm{~m},-3 \mathrm{~m}$, $5 \mathrm{~m})$, respectively. authors using the old method with results of the verification method based on computer prediction described in this paper. These comparisons are intended to prove the superiority of the sound source verification obtained with the new method compared to the previous one, in addition to its greater flexibility and lower cost.

The authors have previously collected data outdoors from various sound sources using the method of fictitious surfaces in the arrangement required by the ISO standard. The goal of this section is to find agreement between actual in situ measurements and predictions made using the new method. The two major difficulties involved in comparing a computer scenario to reality are finding the distance from the source to the ground and the absorption coefficient of this ground surface. Both factors have been obtained in this section by trial and error, though always based on realistic values. Several comparisons between the two methods have been performed, and all have shown very reasonably consistent results. One of these comparisons is now presented and discussed as an example. Of all the loudspeakers checked in the past and available to be characterised by the new method, the sound source B\&K 4224 is widely used by the authors to measure façade acoustic insulation according to the ISO $140-5$ standard. This study looked at in situ measurement data using the B\&K 4224 loudspeaker positioned horizontally on the ground and aimed at the centre of a fictitious measuring surface with dimensions of $10 \mathrm{~m} \times 4 \mathrm{~m}$. The plan view of the arrangement's coordinate system, reproducing the actual in situ verification, is visualised in Fig. 11. The loudspeaker being tested, the model B\&K 4224 labelled as $\mathrm{S}\left(-30^{\circ}\right)$ in the figure, is placed at the specified coordinates $(4.3 \mathrm{~m},-2.5 \mathrm{~m}, 5 \mathrm{~m})$ and has azimuth angle of $30^{\circ}$. The surface size from which SPL measurements have been taken measures $\Delta x \times \Delta y=10 \mathrm{~m} \times 4 \mathrm{~m}$. The ground is considered to be $29 \mathrm{~cm}$ from the loudspeaker's acoustic centre.

Fig. 12 shows the maximum level differences in the one-third octave bands from $100 \mathrm{~Hz}$ to $5 \mathrm{kHz}$ on the façade measuring $10 \mathrm{~m} \times 4 \mathrm{~m}$ for the B\&K 4224 loudspeaker arrangement shown in Fig. 11. Three different cases are compared. In Fig. 12a computer predictions following the proposed procedure with direct sound field estimation over the surface are visualised. Fig. 12b depicts computer predictions considering the acoustic reflection in the ground, with the absorption coefficient adjusted to $\alpha=0.5$. Fig. $12 \mathrm{c}$ shows the results from the in situ outdoor verification with real measurements.

Fig. 13 shows three maps and histograms of sound pressure level distribution on the $10 \mathrm{~m} \times \mathbf{4 m}$ façade in the octave band of 

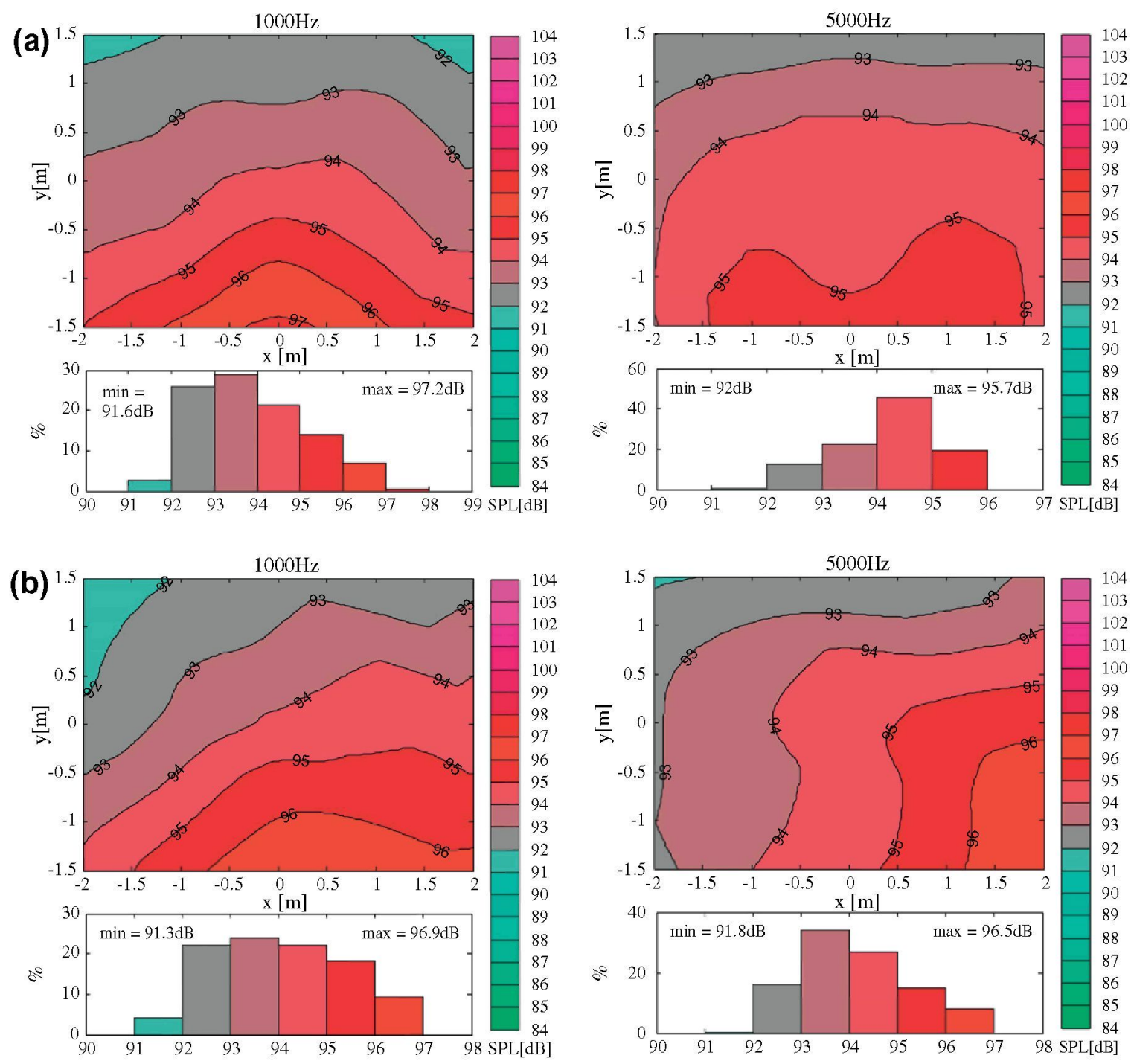

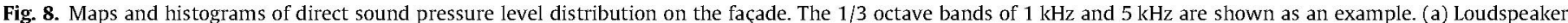
from point $S_{+}\left(-90^{\circ}\right)$ in Fig. 7. (b) Loudspeaker from point $S_{-}\left(-36.9^{\circ}\right)$ in Fig. 7.

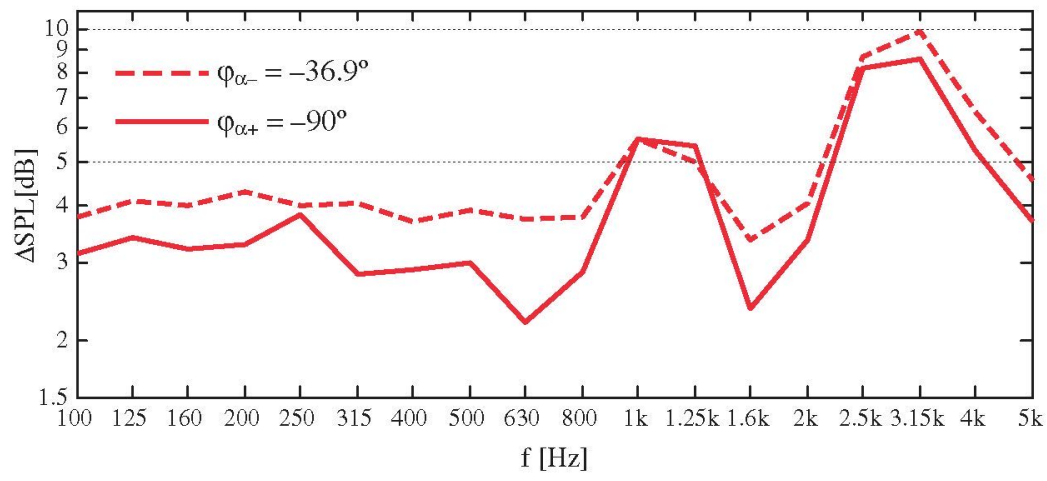

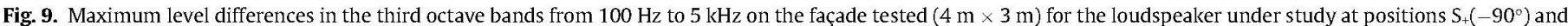

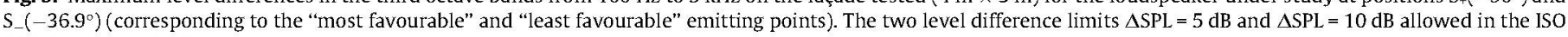
$140-5$ are depicted here.

$1 \mathrm{kHz}$. Fig. 13a shows computer prediction using EASE following the proposed procedure, considering only the direct acoustic incidence from the loudspeaker. Fig. 13b displays the same predictions as in (a), but considering the reflection in the ground, with the absorption coefficient adjusted to $\alpha=0.5$. Fig. $13 \mathrm{c}$ represents the actual measurement coming from in situ verification with outdoor SPL measurements. In all three cases, the loudspeaker $\mathrm{S}\left(-30^{\circ}\right)$ representing model B\&K 4224 was placed as in Fig. 11 at coordinates 


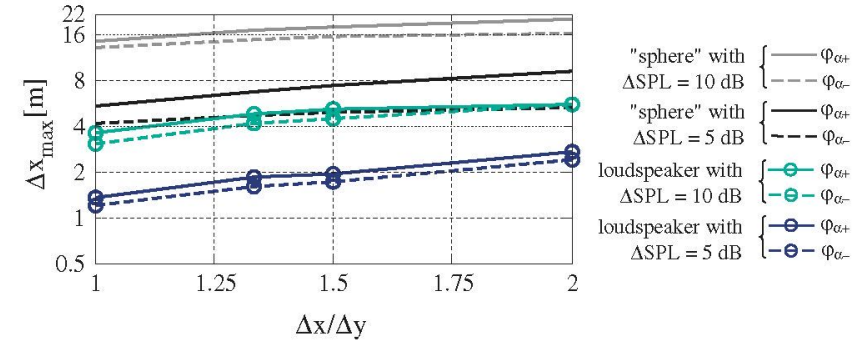

Fig. 10. Maximum horizontal dimension of the façade $\Delta x_{\max }$ verifying ISO $140-5$, either with the $\triangle \mathrm{SPL}=5 \mathrm{~dB}$ or $\triangle \mathrm{SPL}=10 \mathrm{~dB}$ criterion, considering one-third octave bands up to $5 \mathrm{kHz}$ and shown compared to the façade aspect ratio $\Delta x / \Delta y$. The maximum dimensions obtained with a reference omnidirectional loudspeaker called a "sphere" are represented along with the tested loudspeaker in this graph. The azimuth angle of the "most favourable" position of the loudspeaker is the same for all aspect ratios, $\varphi_{\alpha+}=-90^{\circ}$. The azimuth angle for the "least favourable" position of the loudspeaker varies with the façade aspect ratio, resulting in $\varphi_{\alpha_{-}}=-45^{\circ},-36.9^{\circ}$,

$-33.7^{\circ}$ and $-26.6^{\circ}$ for $\Delta x / \Delta y=1,4 / 3,3 / 2$ and 2 , respectively. In all cases, the loudspeaker is placed horizontally $5 \mathrm{~m}$ from the facade and aimed at its centre.

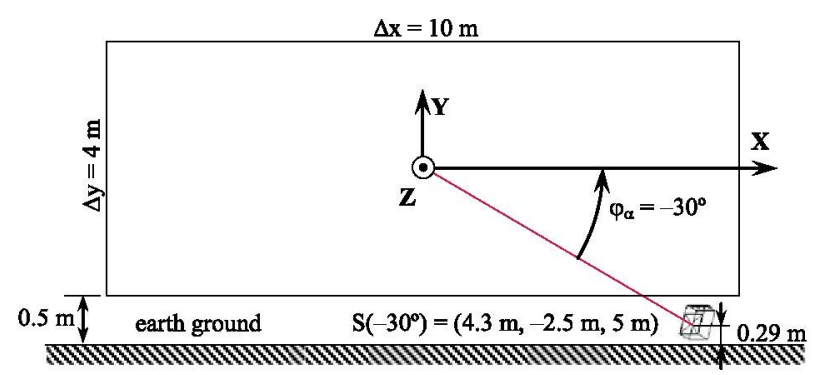

Fig. 11. Plan view in the proposed coordinate system of the loudspeaker being tested, model B\&K 4224, emitting $5 \mathrm{~m}$ from the fictitious façade with size $\Delta x \times \Delta y=10 \mathrm{~m} \times 4 \mathrm{~m}$. The emission point is called $\mathrm{S}\left(-30^{\circ}\right)$ (azimuth angle of $30^{\circ}$ ) and is placed at coordinates $(4.3 \mathrm{~m},-2.5 \mathrm{~m}, 5 \mathrm{~m})$. The ground is located $29 \mathrm{~cm}$ from the acoustic centre of the loudspeaker.
$(4.3 \mathrm{~m},-2.5 \mathrm{~m}, 5 \mathrm{~m})$. The data shown in the maps have an accuracy of $1 \mathrm{~m}$.

Important conclusions can be drawn from Figs. 12 and 13. First, the in situ outdoor measurements of Fig. 13c seem to suffer the effects of ground reflection [10-12], as the effects are also reflected in the computer simulation of Fig. 13b. Therefore, these measurements are not useful for verifying the loudspeaker according to the ISO 140-5 standard because the free field condition is mandatory to accomplish this verification. Although ground reflection in actual measurements could be mitigated at mid- to high-range frequencies by incorporating an absorbent material between the sound source and the floor (a procedure that was not implemented in the measurements shown in Fig. 13c), it is very difficult to absorb this ground reflection at mid- to low-range frequencies (the effect of reflections between $250 \mathrm{~Hz}$ and $1250 \mathrm{~Hz}$ is present when comparing Fig. 12a with Fig. 12b). The second conclusion to be made is that computer simulations can reproduce quite accurately what happens in a simple case such as this one when considering only ground reflection. Although adjustments were made in the simulation process by trying to fix the distance from the loudspeaker acoustic centre to the floor and the absorption coefficient of this floor material at all frequencies of interest, a strong correlation between simulation and actual measurements was found in all loudspeaker simulations (including those not shown here), appearing as the standing waves shown in Fig. 13 for low- to mid-range frequencies.

It can be concluded that the proposed method is superior to the in situ outdoor measurement procedure. This method more reliably determines how the loudspeaker acoustic coverage behaves in free field conditions, as required by the ISO standard. The method is also cheaper and more flexible because a speaker can be verified only once and used later as needed to prove its effect on façades of multiple sizes and orientations in acoustic free field conditions.

Table 1

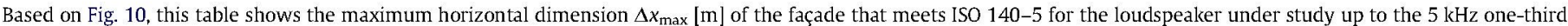

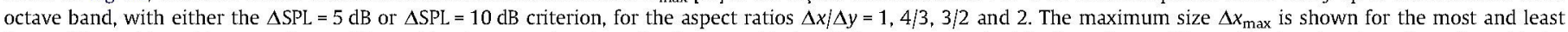

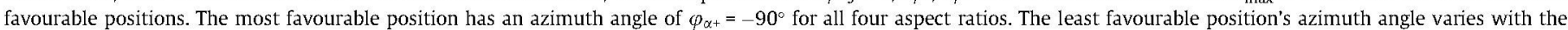
aspect ratio, being $\varphi_{\alpha-}=-45^{\circ},-36.9^{\circ},-33.7^{\circ}$ and $-26.6^{\circ}$ for $\Delta x / \Delta y=1,4 / 3,3 / 2$ and 2 , respectively.

\begin{tabular}{|c|c|c|c|c|c|c|c|c|}
\hline \multirow{2}{*}{$\begin{array}{l}\Delta x / \Delta y= \\
\Delta S P L=\end{array}$} & \multicolumn{2}{|l|}{1} & \multicolumn{2}{|l|}{$4 / 3$} & \multicolumn{2}{|l|}{$3 / 2$} & \multicolumn{2}{|l|}{2} \\
\hline & $5 \mathrm{~dB}$ & $10 \mathrm{~dB}$ & $5 \mathrm{~dB}$ & $10 \mathrm{~dB}$ & $5 \mathrm{~dB}$ & $10 \mathrm{~dB}$ & $5 \mathrm{~dB}$ & $10 \mathrm{~dB}$ \\
\hline$\varphi_{\alpha+}=$ & $-90^{\circ}$ & $-90^{\circ}$ & $-90^{\circ}$ & $-90^{\circ}$ & $-90^{\circ}$ & $-90^{\circ}$ & $-90^{\circ}$ & $-90^{\circ}$ \\
\hline$\Delta x_{\max }=$ & $1.35 \mathrm{~m}$ & $3.65 \mathrm{~m}$ & $1.85 \mathrm{~m}$ & $4.85 \mathrm{~m}$ & $1.95 \mathrm{~m}$ & $5.20 \mathrm{~m}$ & $2.7 \mathrm{~m}$ & $5.5 \mathrm{~m}$ \\
\hline$\varphi_{\alpha-}=$ & $-45^{\circ}$ & $-45^{\circ}$ & $-36.9^{\circ}$ & $-36.9^{\circ}$ & $-33.7^{\circ}$ & $-33.7^{\circ}$ & $-26.6^{\circ}$ & $-26.6^{\circ}$ \\
\hline$\Delta x_{\max }=$ & $1.20 \mathrm{~m}$ & $3.05 \mathrm{~m}$ & $1.60 \mathrm{~m}$ & $4.20 \mathrm{~m}$ & $1.70 \mathrm{~m}$ & $4.50 \mathrm{~m}$ & $2.4 \mathrm{~m}$ & $5.5 \mathrm{~m}$ \\
\hline
\end{tabular}

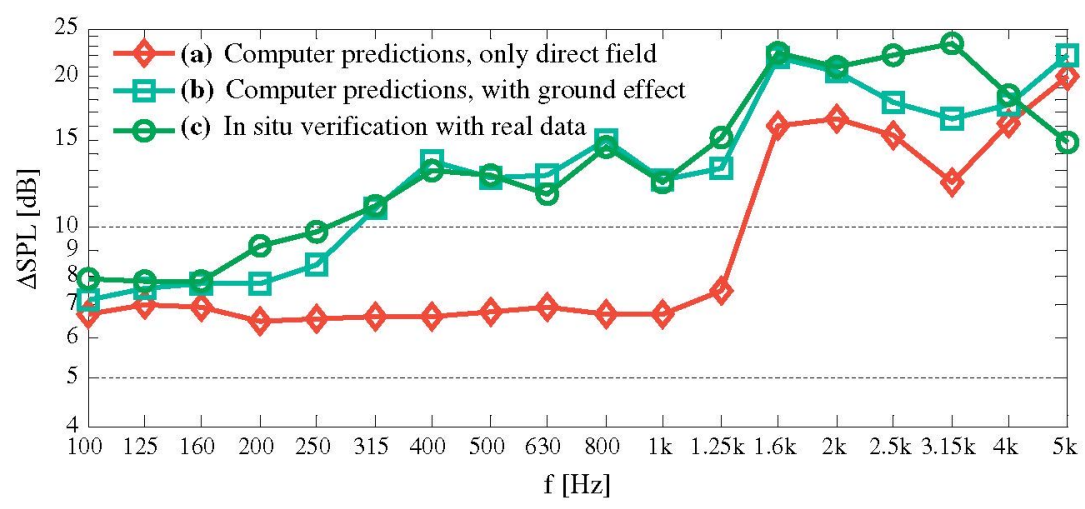

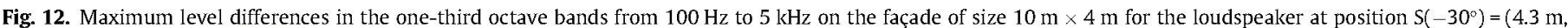

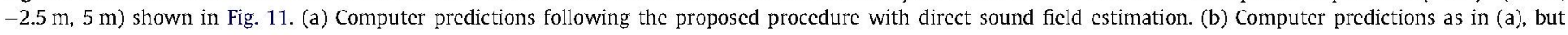
considering the reflection in the ground with the absorption coefficient adjusted to $\alpha=0.5$. (c) In situ verification with real measurements. 

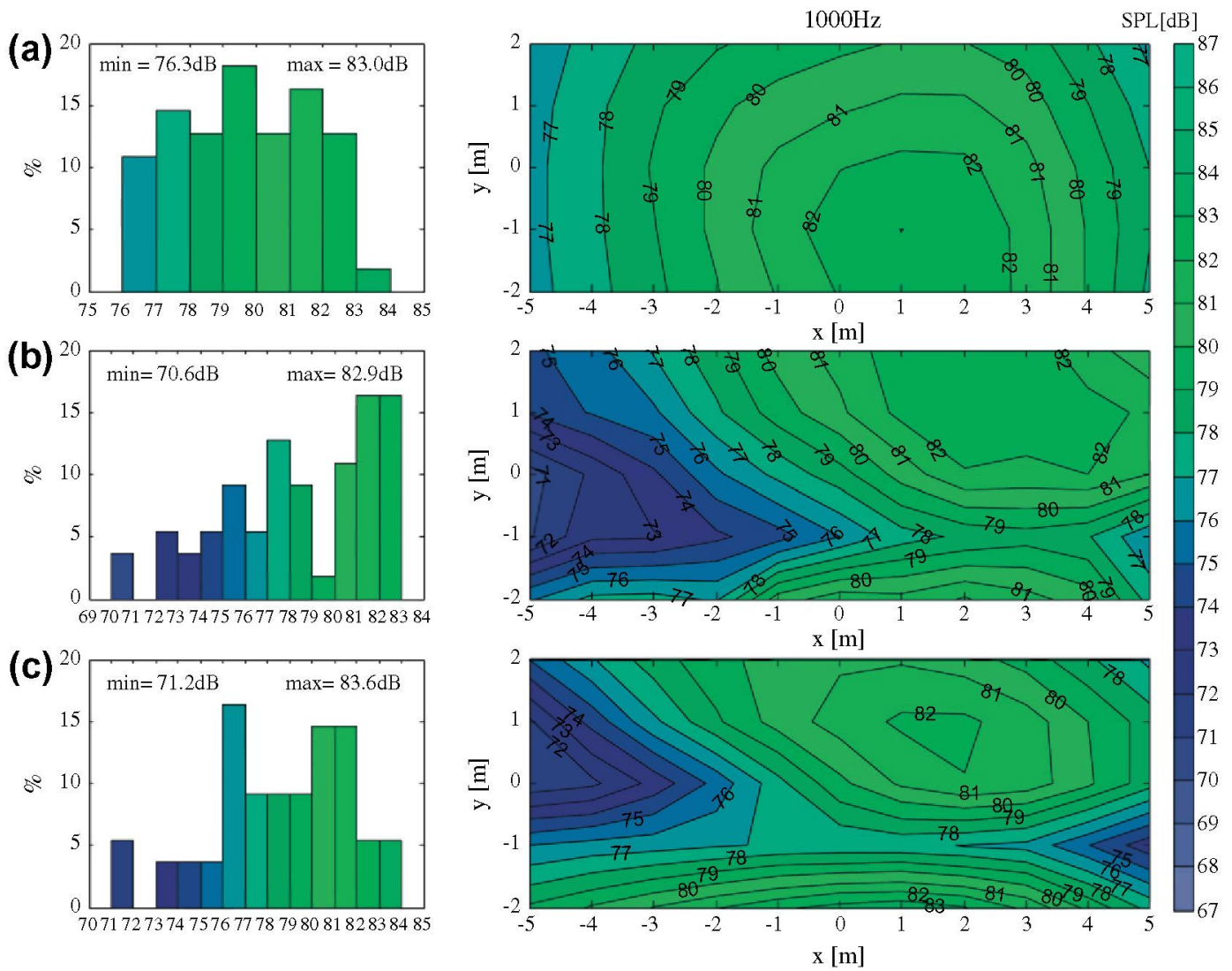

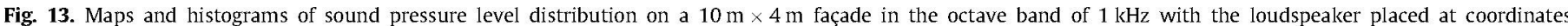

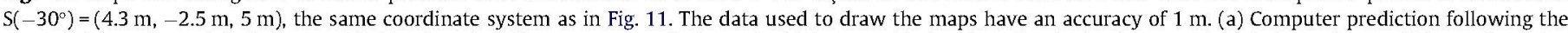

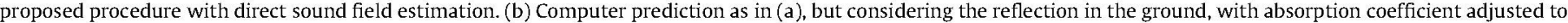
$\alpha=0.5$ and the loudspeaker acoustic centre placed $29 \mathrm{~cm}$ from the floor. (c) In situ verification with real measurements.

\section{Conclusions}

This paper has presented a new verification procedure for sound source coverage based on the ISO $140-5$ requirements. This procedure has been successfully used by the authors with the loudspeakers utilised for their current and past façade insulation measurements. The method consists of the electroacoustic characterisation of the sound source in laboratory free field conditions (an anechoic room) and the subsequent computer simulation prediction of the free sound field radiated on a façade. Coverage requirements for the source are difficult to validate outdoors because of the need to achieve acoustic-free field conditions. Prior to using the proposed method, the authors had verified sound sources outdoors in as free a sound field as possible.

As demonstrated in the paper, ensuring free field condition outdoors is difficult because ground reflection on the façade surface cannot be completely avoided. In addition, outdoor measurements are usually not accurate enough because of the difficulty of measuring large surfaces. Each sound source position also requires the use of a costly measurement process in this scenario. In contrast, the proposed method is more flexible and repeatable; it allows for high resolution predictions and the testing of multiple sound source positions and façades of varying sizes. The effect of unwanted reflections can be fully avoided, and the maximum size of the façade applicable to each sound source can be calculated.

These findings may assist in thinking about the usefulness of the ISO standard requirements for loudspeaker sound coverage on a façade. Noting that it is not clear if level differences on the façade have a large influence on insulation measurements anyway [13], it might be better to specify the maximum angle of directivity coverage of the sound source according to the maximum angle at which the façade is "seen" by the loudspeaker, a point stated in the geometric study in [6]. These suggestions should help to improve future versions of the ISO 140-5 standard regarding the insulation of façades.

\section{References}

[1] ISO 140-5:1998. Acoustics-measurement of sound insulation in buildings and of building elements. Part 5: field measurements of airborne sound insulation of façade elements and façades.

[2] Rasmussen B, Rindel JH. Sound insulation between dwellings - descriptors applied in building regulations in Europe. Appl Acoust 2010;71(3):171-80.

[3] Rasmussen B. Sound insulation between dwellings - requirements in building regulations in Europe. Appl Acoust 2010;71(4):373-85.

[4] ISO/IEC 17025:2005. General requirements for the competence of testing and calibration laboratories.

[5] Sánchez Bote JL, Gómez Alfageme, IJ. Procedimiento para la medición y verificación de la directividad y la cobertura de una fuente sobre un elemento de fachada de acuerdo a la norma ISO 140-5. In: Proc. Tecniacústica 2011. Cáceres (Spain); October 26-28, 2011. 11 pp. (in Spanish).

[6] Sánchez Bote JL, Pedrero González A, Gómez Alfageme IJ. Influence of loudspeaker directivity and measurement geometry on direct acoustic levels over façades for acoustic insulation tests with the International Standard ISO 140-5. Appl Acoust 2012;73(4):440-53.

[7] EASE. Enhanced acoustic simulator for engineers. <http://ease.afmg.eu/>

[8] Feistel S, Ahnert W. Modeling of loudspeaker systems using high-resolution data. J Audio Eng Soc 2007:55:571-97.

[9] Feistel S, Ahnert W, Hughes C, Olson B. Simulating the directivity behavior of loudspeakers with crossover filters. Paper 7254, 123rd convention, Audio Engineering Society. New York, USA; October 5-8, 2007.

[10] Hopkins C, Lam Y. Sound fields near building facades - comparison of finite and semi-infinite reflectors on a rigid ground plane. Appl Acoust 2009;70(2):300-8.

[11] Berardi U, Cirillo E, Martellotta F. Interference effects in field measurements of airborne sound insulation of building facades. Noise Control Eng J $2011 ; 59(2): 165-76$. 
[12] Berardi U, Cirillo E, Martellotta F. Measuring sound insulation of building facades: interference effects and reproducibility. In: Proc. inter noise 2010. Lisbon (Portugal); June 13-15, 2010. $10 \mathrm{pp}$.
[13] Pedrero González A, Sánchez Bote JL, Ulin Nabatov V, Diaz Sanchidrián C. Influence of loudspeaker directivity on the measurement uncertainty of the acoustic testing of façades. In: Proc. inter noise 2011. Osaka (Japan); September 4-7, 2011. 6 pp. 